\title{
A entrevista social no processo de avaliação geriátrica ampla
}

\author{
The social interview in the comprehensive geriatric assessment process
}

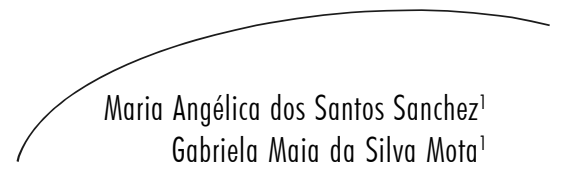

\section{Resumo}

As novas diretrizes do pacto de gestão fortalecem as ações de atenção à saúde do idoso a partir do momento em que se torna uma das prioridades do Ministério da Saúde. Além disso, a Política Nacional de Saúde da Pessoa Idosa preconiza uma atenção integral e integrada com ênfase em abordagens multidimensionais que buscam resgatar ou preservar a autonomia e independência do sujeito que envelhece. Um novo paradigma na atenção emerge trazendo a avaliação geriátrica ampla, realizada por uma equipe multidisciplinar, como uma prática essencial no rastreamento e identificação dos variados problemas que acometem o indivíduo idoso. Nesta modalidade de avaliação é fundamental um olhar direcionado aos problemas sociais que podem influenciar no restabelecimento da saúde ou no aparecimento de novas doenças. A entrevista social é um instrumento técnico que fornecerá ao profissional subsídios para realizar uma avaliação que possa gerar uma intervenção adequada a partir do conhecimento das possibilidades e limitações dos usuários que buscam atenção em unidades geriátricas. O objetivo deste trabalho é tecer algumas considerações acerca da avaliação social no processo de avaliação geriátrica ampla. É também um esforço preliminar de contribuir com o fazer profissional do assistente social, visto que as produções teóricas neste campo são escassas e as questões sociais se constituem em elementos essenciais para o estabelecimento de condutas terapêuticas eficazes.

Universidade do Estado do Rio de Janeiro. Faculdade de Ciências Médicas, Departamento de Pós-Graduação em Ciências Médicas. Rio de Janeiro, RJ, Brasil

Correspondência / Correspondence

Maria Angélica Sanchez

CIPI-UERJ

Avenida Mal. Rondon 381, $2^{\circ}$ andar - São Francisco Xavier

20950-000 - Rio de Janeiro, RJ, Brasil

E-mail: asanchezrj@terra.com.br

Palavras-chave:

Política de Saúde.

Serviços de Saúde para Idosos.

Avaliação Geriátri-

ca. Equipe de

Assistência ao

Paciente. Entrevis-

ta. Idoso. Condi-

ções Sociais.

Assistência

Centrada no

Paciente Avaliação

Social. Serviço

Social. 
Abstract

The new guidelines of the pledge management strengthen actions towards the elderly care now that it has become one of the Health Ministery's priorities. Furthermore, the National Policy of Elderly prescribes a thorough and integrated care with emphasis on the multidimensional approaches that aim at rescuing or preserving both the autonomy and independence of the aging subject. A new paragon in the care appears bringing the comprehensive geriatric assessment, which is accomplished by a multidisciplinary team, as an essential praxis of both tracking and identifying the numerous illnesses to which the elderly is susceptible to. In this kind of assessment, it is fundamental to look into the social issues that might either retard the elderly's recuperation or bring about new maladies. The social interview is a technical instrument that will provide the professional with resources for an assessment that will entail an adequate intervention from

Key words: Health Policy. Health Services for the Aged. Geriatric Assessment. Patient Care Team. Interview. Aged. Social

Conditions. PatientCentered Care. Social Assessment. Social Work. Comprhensive Geriatric Assessment the knowledge of the possibilities and limitations of the patients coming to geriatric facilities. This work aims at taking under consideration the social assessment withing the range of the comprehensive geriatric assessment. It is also an effort to contribute to the praxis of the social worker, given that theory in this field is rare and social issues are central to the establishment of efficient therapeutic procedures.

INTRODUÇÃO

Apesar de a discussão acerca do envelhecimento da população brasileira ter entrado em pauta há algumas décadas, somente com a aprovação do pacto de gestão em fevereiro de 2006 a saúde do idoso entrou no rol das prioridades do Ministério da Saúde. ${ }^{1}$

Alguns desdobramentos da normatização da Portaria 399 do Ministério da Saúde já são observados. A Política Nacional de Saúde da Pessoa Idosa, por exemplo, estimula ações com ênfase em uma abordagem global, interdisciplinar e multidimensional, levando em consideração a interação entre os fatores físicos, psicológicos e sociais a influenciar a saúde do idoso.,
$\mathrm{O}$ atendimento destinado ao idoso requer uma abordagem multidisciplinar, e a intervenção deve ser sempre precedida de uma avaliação abrangente, levando em consideração que, na maioria das vezes, somente uma equipe multidisciplinar integrada é capaz de reabilitar a capacidade funcional de uma pessoa idosa. ${ }^{3}$

A avaliação geriátrica ampla contempla um conjunto de informações sobre os estados clínico, psíquico, funcional e social da pessoa idosa. Trata-se de uma busca ativa de problemas que podem gerar situações complexas, sobretudo em idosos frágeis. Este tipo de avaliação, realizada por uma equipe multidisciplinar, permite um enfoque adequado dos complexos e variados problemas dos idosos. $^{4}$ 
Para que uma avaliação dessa natureza forneça subsídios para um plano terapêutico adequado, faz-se necessário que as informações colhidas apresentem um retrato de todos os aspectos clínicos, além de uma avaliação das condições sociais do indivíduo. Neste sentido, a avaliação social é parte importante neste processo.

Dentre as atribuições do assistente social, a avaliação social é realizada com o objetivo de conhecer a realidade do usuário e suas interações no contexto em que está inserido. ${ }^{5}$ É nesse momento que são identificadas condições sociais que, porventura, podem interferir no restabelecimento de sua saúde. Para tanto, o profissional utiliza como instrumento a entrevista social. ${ }^{6}$

Este artigo tem como objetivo abordar alguns aspectos gerais, importantes na utilização da entrevista como um instrumento técnico na prática do serviço social, bem como sua aplicabilidade no setor saúde, sobretudo na avaliação social no campo da atenção ao idoso.

\section{A entrevista na prática do Serviço Social}

A entrevista é um dos instrumentais mais utilizados na prática do Serviço Social. Ela tem como objetivo precípuo a coleta de dados para conhecer o usuário e a sua demanda, bem como reunir condições efetivas de intervenção profissional. Neste sentido, a entrevista possibilita ao assistente social o conhecimento das reais demandas aparentes e implícitas. ${ }^{7}$
A entrevista caracteriza-se por sua relação dialógica, sendo muito utilizada no espaço profissional e tem como proposta inicial o estabelecimento de uma aproximação do profissional com o usuário no intuito de compreender o que ele deseja e solicita do ambiente de intervenção profissional. Pode ser construída através de um roteiro estruturado com perguntas formuladas levando em consideração a área na qual o profissional está atuando. ${ }^{8}$

É importante que o assistente social deixe transparente, logo de inicio, para o usuário qual é o objetivo daquele procedimento técnico, e em que sentido ele intervirá na problemática levantada, bem como a finalidade do uso das informações coletadas, demonstrando o caráter sigiloso daquele espaço profissional. ${ }^{9}$ Ademais, cabe ao profissional uma atenção especial ao momento da entrevista, não emitindo opiniões decorrentes de sua avaliação pessoal, não julgando e não tomando atitudes preconceituosas; caso contrário, o processo da entrevista pode ser inteiramente comprometido. ${ }^{9}$

A maioria das entrevistas, independente de qual seja o seu tipo, estruturada ou não, tem como principal objetivo conhecer o problema trazido, compreendendoo e solucionando-o eficazmente. ${ }^{9}$ Desta forma, a entrevista favorecerá o levantamento de questões importantes para, posteriormente, serem orientadas ao longo do processo de trabalho. É uma técnica cuja dimensão de coleta de dados permite traçar um perfil da população atendi- 
da, além de conhecer as demandas que são fundamentais para a intervenção profissional. ${ }^{10}$

A entrevista é um instrumento técnicooperativo que não deve ser visto como um fim em si mesmo, mas um meio que favorecerá, num processo mais amplo, a avaliação social. $^{5}$

Com este instrumental é possível avaliar as demandas do usuário do serviço e da situação relatada por ele para que futuramente seja realizada uma avaliação mais abrangente com todos os dados coletados para uma possível elaboração de um plano de intervenção para o problema apresentado. ${ }^{5}$

É importante, porém, que o profissional tenha conhecimento do espaço institucional em que está atuando, bem como, dos recursos disponíveis, principalmente, na prática profissional do assistente social, a fim de poder concretizar as demandas do usuário. ${ }^{9}$

Contudo, a entrevista estruturada não deve ser uma camisa de força para o profissional, a ponto de impedi-lo de ir além do que está estabelecido, se assim julgar ser importante para aprofundar alguns aspectos que não constem no guia da entrevista. Ela nada mais é que um roteiro que auxilia na organização das idéias para um maior entendimento da situação levantada pelo usuário. ${ }^{8}$

O importante é ter a clareza de que a prática do assistente social não deve se alicerçar em uma atividade rotineira de coleta de informações. Silva ${ }^{10}$ alude à importância de um momento de reflexão após a entrevista que possa embasar a sua ação; ou ainda concretizar a sistematização da prática, uma vez que isto possibilita o aprimoramento do fazer profissional no espaço institucional.

Por outro lado, a entrevista é o momento em que o profissional lança mão dos aspectos educativo e reflexivo para a instrumentalização da população usuária dos serviços, com relação aos seus direitos e deveres como cidadãos. ${ }^{7}$ Neste sentido, a socialização da informação constitui instrumento de indagação e ação onde o aspecto educativo é direcionado para a transformação das condições que podem ser geradoras dos problemas vivenciados pelos usuários. ${ }^{7}$

0 papel da avaliação social no protocolo de avaliação geriátrica ampla

Kane $^{6}$ traz para reflexão duas vertentes acerca da avaliação social. Sob o ponto de vista $d a$ equipe multidisciplinar, trata-se apenas de mais uma dimensão do usuário que deve ser avaliada. No entanto, que sob a óptica das ciências sociais, pode ser vista como uma avaliação abrangente e complexa que contempla os múltiplos problemas apresentados pelo idoso.

Por ser a avaliação geriátrica ampla uma avaliação multidimensional, compreende em sua estrutura uma avaliação do contexto social do idoso obtida através de uma entrevista que, em um ambiente multidisci- 
plinar, fica sob a responsabilidade do assistente social. Este analisa amplamente as circunstâncias sociais e sugere que intervenções ou alternativas são necessárias para a resolução do problema apresentado pelo usuário.

A prática gerontológica efetiva demanda a realização de uma avaliação minuciosa das condições sociais do idoso em acompanhamento, buscando analisar se tais condições estão influenciando negativamente o restabelecimento da saúde ou se são responsáveis pelo surgimento de novos problemas. Os idosos, por apresentarem múltiplas queixas que interagem com as condições físicas, emocionais e cognitivas, talvez sejam mais sensíveis às alterações advindas do ambiente social. ${ }^{6}$

Apesar de a avaliação social permitir, a partir de uma ação integrada à dos demais profissionais, uma visão integral do sujeito que adoece e favorecer maior compreensão das implicações sócio-culturais na saúde, é ainda pouco desenvolvida no processo de trabalho do assistente social. ${ }^{11}$

Ainda são escassas no Brasil as produções teóricas sobre a avaliação social no campo da gerontologia, ao contrário de outros países que dispõem de um manancial de instrumentos que avaliam, separadamente, diferentes dimensões das condições sociais da pessoa idosa como, por exemplo: rede de suporte social; ambiente do domicílio; espiritualidade; situação econômica; saúde sexual e sobrecarga do cuidador.

Ainda que não tenhamos disponíveis tais instrumentos, faz-se importante que essa ava- liação seja composta por questões relevantes para a análise de problemas e para a intervenção nos espaços de atenção geriátrica.

Dimensões relevantes para a avaliação social da pessoa idosa

Em uma avaliação social são colhidas muitas informações que permitem ao profissional traçar o perfil do idoso em atendimento e conhecer um pouco da sua dinâmica familiar. Mesmo que o guia de entrevista abarque vários aspectos, consideramos importante destacar três dimensões que devem ser alvo constante na avaliação.

\section{A Rede Social}

Uma rede social adequada é uma das condições fundamentais para o sucesso do plano terapêutico. Kane ${ }^{6}$ define a rede social como a teia de relações e contatos na vida de um indivíduo.

A rede social, em um nível microscópico, constitui-se na rede social pessoal e possui certas funções a serem desempenhadas por seus integrantes: oferecer companhia; destinar apoio emocional; servir como guia de conselhos e de regulação social; fornecer ajuda material e facilitar o acesso a novos contatos. ${ }^{12}$

É fundamental conhecer as características estruturais desta rede, como por exemplo, o número de pessoas que a compõe, como estas pessoas se relacionam, e como essa rede esta composta. ${ }^{12}$ 


\section{Suporte Social}

O suporte social refere-se à assistência objetiva destinada ao idoso e divide-se em formal - aquele oferecido pelas instituições governamentais, e o suporte informal - destinado pela família. O suporte social é definido como a assistência objetiva fornecida pela rede social, levando-se em consideração a impressão subjetiva de quem o recebe. ${ }^{6}$

O suporte destinado a indivíduos por seus familiares é mais importante na velhice do que em qualquer outra fase da vida, com exceção da infância, quando a família tem um papel fundamental. ${ }^{13}$

No Brasil, o suporte social é fornecido, em grande parte, pela família. As políticas de atenção destinadas à população que envelhece caminham timidamente na implementação de ações que atendam satisfatoriamente ao idoso e a seu cuidador.

Para avaliar o suporte social, o profissional pode utilizar questionários e escalas, ou entrevistas semi-estruturadas que incluam informações sobre o tipo de suporte fornecido, quem o fornece e como este é recebido. ${ }^{13}$

Mais importante que avaliar o suporte social no seu aspecto quantitativo, é avaliar se o indivíduo recebe o mínimo de suporte. Para isto, Kane $e^{6}$ sugere três aspectos a serem avaliados: (1) com quem a pessoa pode contar quando precisa de ajuda; (2) a periodicidade do contato que mantém com seus parentes mais próximos; (3) a convivência com amigos e a periodicidade de contato com eles, sendo também importante avaliar a disponibilidade de as pessoas fornecerem esse suporte.

\section{A situação econômica}

Os fatores econômicos têm um impacto relevante na vida de uma pessoa. Eles são determinantes das condições de moradia, da saúde nutricional e da obtenção de medicamentos, entre outros. Gallo et al. ${ }^{13}$ comentam que a conduta terapêutica adotada por uma equipe pode ser completamente alterada pela pessoa em decorrência da precariedade de seus recursos financeiros.

A avaliação das condições econômicas é fator preponderante para a elaboração do plano terapêutico para o idoso em acompanhamento. Ela também constitui-se em facilitador para as tomadas de decisões. No entanto, a ausência de recursos muitas vezes impede uma conduta adequada, exercendo efeitos negativos na adesão ao tratamento. Ao conhecer a condição econômica e as possibilidades da rede social do usuário, o profissional deverá articular-se fortemente para viabilizar o acesso aos recursos disponíveis na comunidade.

\section{A Sobrecarga do cuidador}

A discussão sobre o impacto da sobrecarga do cuidador na vida de um idoso dependente é assunto pouco disseminado no Brasil. Ainda que o tema mereça destaque, é notória a ausência de uma política direcionada para este segmento da população. ${ }^{14}$ 
O cuidador de um idoso dependente deve ser visto como um potencial parceiro da equipe. $\mathrm{Na}$ maioria das vezes, porém, ele não escolheu essa tarefa, apenas assume-a por conta das circunstâncias impostas. $^{15}$

Uma das formas de se avaliar o cuidador é a utilização de escalas que possam aferir seus níveis de sobrecarga. Uma delas é a escala de sobrecarga de Zarit, ${ }^{16}$ um instrumento que vem sendo largamente utilizado em todo o mundo. Scazufca ${ }^{17}$ propõe uma versão para uso no Brasil que tem sido utilizada em muitos espaços de atenção geriátrica.

Além da utilização da escala, uma avaliação subjetiva e bem detalhada do cuidador, fornece ao profissional um panorama geral do idoso em acompanhamento e das reais possibilidades de suporte e de adesão ao tratamento.

A avaliação social em uma unidade ambulatorial geriátrica: a prática do serviço social

No ambulatório de uma unidade de assistência geriátrica, localizada em um hospital universitário na cidade do Rio de Janeiro, o Serviço Social atua seguindo os princípios éticos que norteiam o projeto políticoprofissional e em consonância com as diretrizes da prática geriátrico-gerontológica.

O trabalho é desenvolvido com base na prática reflexiva do processo de envelheci- mento e suas conseqüências no âmbito da saúde, da família e da sociedade. Além disso, faz parte do escopo do espaço de atenção um conjunto de orientações sobre o exercício dos direitos da pessoa idosa.

A avaliação social é utilizada em todas as atividades que requeiram a intervenção do serviço social, seja na avaliação geriátrica ampla, seja na avaliação do cuidador de idosos dependentes, ou, ainda, na avaliação de idosos que ingressam nos grupos de promoção de saúde. Cada um desses momentos tem um modelo diferenciado e objetivos específicos descritos a seguir.

A avaliação social inicial é, geralmente, o primeiro contato que o assistente social tem com o usuário. Trata-se da primeira sessão da avaliação geriátrica ampla e tem como objetivo identificar os aspectos sociais que podem influenciar a saúde do idoso ou vice-versa.

Essa avaliação é importante para subsidiar a tomada de decisões na implementação do Plano de Cuidados, pois as observações realizadas na entrevista são essenciais para um planejamento adequado que respeite os limites e não fuja das possibilidades do usuário e de sua rede de suporte.

Ela geralmente ocorre antes da avaliação clínica para possibilitar aos demais integrantes $d a$ equipe um conhecimento da real situação do idoso, permitindo às condutas estabelecidas estarem ao alcance do usuário. Com duração média de quarenta minutos, ela pode variar conforme a necessidade de cada pessoa. 
A maioria das condutas é estabelecida após discussão do caso com a equipe. Entretanto, as situações que requeiram intervenção imediata são discutidas com o idoso e família buscando, em conjunto, alternativas para a situação apresentada.

Uma outra modalidade de entrevista é a avaliação social do cuidador, que tem como objetivo conhecer as demandas da pessoa responsável pelo cuidado direto com o idoso dependente, em decorrência de uma síndrome demencial.

O cuidador é encaminhado ao serviço social pelo médico depois que este informa o diagnóstico à família e apresenta o prognóstico da doença. Nessa avaliação, cabe ao assistente social conhecer a dinâmica familiar, bem como os recursos deste cuidador, para discutir com o mesmo as possibilidades e limitações de sua tarefa. Cabe ao profissional identificar que políticas setoriais atendem às demandas do cuidador, orientando-o quanto ao exercício de seus direitos.

\section{CONSIDERAÇÕES FINAIS}

Embora esteja aumentando o interesse do profissional do serviço social neste campo de atenção, o tema avaliação social no âmbito da assistência geriátrica é pouco estudado e divulgado no Brasil.

Em parte isso se deve à escassez de produções teóricas, com conteúdo mais didático do faz̧ere do agir nos espaços de atenção ao idoso. Apesar de o assistente social ser um profissional importante em uma equipe multidisciplinar, quer pelas possibilidades de uma assessoria, quer pela atenção direta ao usuário do serviço, a profissão ainda carece de recursos para desenvolver um papel determinante, sobretudo em um país cujas políticas setoriais destinadas a este segmento etário ainda são incipientes.

Historicamente, o serviço social é visto como um espaço de resolução de problemas para onde o usuário é encaminhado depois que os demais profissionais não têm mais alternativas. No entanto, é preciso que o profissional do serviço social distinga seu campo de atuação com clareza. Para tanto, faz-se necessária a instauração de um novo fazer, criando possibilidades de intervenção que possam ir ao encontro dos anseios e possibilidades do usuário.

$\mathrm{Na}$ assistência geriátrica, os problemas que acometem o idoso têm forte relação com suas condições sociais. São inúmeras as situações que influenciam negativamente sua saúde e aqui podemos destacar, principalmente, os dilemas da ausência de uma rede de suporte, a precariedade dos recursos toda natureza para grande parte dessa população, e os maustratos advindos de condições adversas a que está exposto.

Portanto, é fundamental que o assistente social tenha habilidade para enfrentar essas questões; caso contrário, ele será um mero colhedor de informações e fornecedor de encaminhamentos. Além disso, o aprofunda- 
mento de pesquisas nessa área é essencial para o incremento de ações inovadoras que possam contribuir para o bom desempenho do assistente social no campo da gerontologia.

\section{REFERÊNCIAS}

1. Brasil. Ministério da Saúde. Portaria 399 G/M de 22 de fevereiro de 2006. Divulga o Pacto pela Saúde - consolidação do SUS e aprova as diretrizes operacionais do referido Pacto. Brasília: Ministério da Saúde; 2006a.

2. Brasil. Ministério da Saúde. Portaria 2528/ GM de 19 de outubro de 2006. Aprova a Política Nacional de Saúde da Pessoa Idosa. Brasília: Ministério da Saúde; 2006b.

3. Pfeifer E. Some basic principles of working with older patients. J Am Geriatr Soc 1985 Jan;33(1): 44-7.

4. NIH, Geriatric Assessment Methods for Clinical Decisionmaking, NIH Consensus Statement, 1987 oct 19-21; 6 (13):1-21.

5. Moreira MPS. A avaliação social do idoso dependente na assistência domiciliar. [dissertação]. São Paulo: Pontifícia Universidade Católica de São Paulo; 2005.

6. Kane RA. Assessment of social function: recomendations for comprehensive geriatric assessment. In: Rubenstain L.Z, Wieland D. Benrnabei R, editors. Geriatric Assessment Tecnology: The state of the Art. New York: Spring Publish; 1995. p. 91-110

7. Vasconcelos AM. Serviço Social e prática reflexiva. Revista Em Pauta1997; 10:131- 81.

8. Richardson RJ. "Entrevista”. In: Pesquisa social: métodos e técnicas. 2 ed. São Paulo: Atlas; 1989. p. 160-72.
9. Garret, A. A entrevista: seus princípios e métodos. Rio de Janeiro: Agir; 1991. p.15- 116.

10. Silva JAP. O papel da entrevista social na prática do Serviço Social. Revista Em pauta 1995.

11. Assis M. A avaliação social na atenção integral à saúde do idoso.. In: Anais do IX Congresso Brasileiro de Assistentes Sociais, 1998, Goiânia. Caderno de Comunicações do IX Congresso Brasileiro de Assistentes Sociais, 1998. v. 1.p. 333-5

12. Sluzki CE. A rede social na prática sistêmica: alternativas terapêuticas. São Paulo: Casa do Psicólogo; 1997.

13. Gallo JJ, Fulmen T, Paveza GJ, Reichel W. Handbook of Geriatric Assessment . Gaithrsburg, Maryland: Aspen; 2000.

14. Veras RP, et al. Avaliação dos gastos com o cuidado do idoso com demência. Revista de psiquiatria clìnica 2007; 34 (1): 5-12.

15. Karsch, UM. Envelhecimento com dependência: revelando cuidadores. São Paulo: Educ; 1998

16. Zarit SH, Reever KE, Bach-Peterson J. Relatives of impaired elderly: correlates of feelings of burden. Gerontologist 1980; 20: 649-55.

17. Scazufca M. Brasilian version of the Burden Interview scale for the assessment of burden of care in cares of people with mental illness. Revista brasileira de psiquiatria 2000; 24(1):12-7. 
1 